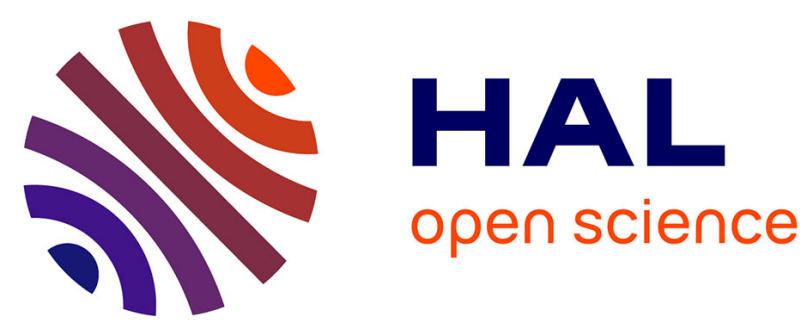

\title{
Structure and Magnetic Properties of Nd0.88MnO3 Films Grown on SrTiO3 (100) Substrates by Liquid-Injection MOCVD
}

Nejib Ihzaz, Michel Boudard, Mohamed Oumezzine

\section{- To cite this version:}

Nejib Ihzaz, Michel Boudard, Mohamed Oumezzine. Structure and Magnetic Properties of Nd0.88MnO3 Films Grown on SrTiO3 (100) Substrates by Liquid-Injection MOCVD. Journal of Electronic Materials, 2020, 49 (10), pp.6055-6060. 10.1007/s11664-020-08350-0 . hal-03367606

\section{HAL Id: hal-03367606 https://hal.science/hal-03367606}

Submitted on 6 Oct 2021

HAL is a multi-disciplinary open access archive for the deposit and dissemination of scientific research documents, whether they are published or not. The documents may come from teaching and research institutions in France or abroad, or from public or private research centers.
L'archive ouverte pluridisciplinaire HAL, est destinée au dépôt et à la diffusion de documents scientifiques de niveau recherche, publiés ou non, émanant des établissements d'enseignement et de recherche français ou étrangers, des laboratoires publics ou privés. 


\title{
Structure and Magnetic Properties of $\mathrm{Nd}_{0.88} \mathrm{MnO}_{3}$ Films Grown on $\mathrm{SrTiO}_{3}$ (100) Substrates by liquid-injection MOCVD
}

\author{
Nejib Ihzaz $^{1,2 *}$, Michel Boudard ${ }^{3}$, Mohamed Oumezzine ${ }^{1}$ \\ ${ }^{1}$ Laboratoire de Physico-Chimie des Matériaux, Département de Physique, Faculté des \\ Sciences de Monastir, 5019 Monastir, Tunisia \\ ${ }^{2}$ Institut Supérieur des Sciences Appliquées et de Technologie de Gabès, Rue Amor Ben El \\ Khatab, 6029 Gabès, Tunisia \\ ${ }^{3}$ LMGP, MINATEC Bâtiment INPG, UMR 5628 CNRS/INPG, 3 Parvis Louis Néel, BP 257, \\ 38016 Grenoble Cedex 1, France
}

\begin{abstract}
:
We have investigated the structure and magnetic properties of the $\mathrm{Nd}_{0.88} \mathrm{MnO}_{3}$ (NMO) perovskite thin-film layers by liquid-injection MOCVD tried on $\mathrm{SrTiO}_{3}$ (STO) (100) substrates. The structural examinations showed that the growing film was amorphous. It changed to the crystalline phase after being annealed at $900{ }^{\circ} \mathrm{C}$ in the air for $24 \mathrm{~h}$. A minor secondary phase identified as $\mathrm{Mn}_{3} \mathrm{O}_{4}$ appears. The film is subjected to in-plane (IP) tensile stress leading to a contraction of the film out of the plane (OOP) lattice parameters. The orientation relationships for the film structure is described near the interface as $[10 \overline{1}] \mathrm{NMO} / /[100] \mathrm{STO}$ and $[010] \mathrm{NMO} / /[001] \mathrm{STO}$. The NMO thin film showed ferromagnetic ordering with a transition temperature $\mathrm{T}_{\mathrm{C}}=85 \mathrm{~K}$. The large bifurcation between ZFC and FC curves with a cusp at $T_{f}=53 \mathrm{~K}$; and an irreversible temperature $T_{\text {irr }}=48 \mathrm{~K}$ proves the spin-glass behavior with long-range ferromagnetic order $\left(\mathrm{Mn}^{3+}-\mathrm{O}^{2-}-\mathrm{Mn}^{4+}\right)$ coexists in NMO thin films. The magnetic easy axis corresponded to the surface normal.
\end{abstract}

Keywords: Manganite, Grown thin film, liquid-injection MOCVD, X-ray diffraction, out-ofplane texture, Magnetization, Spin-Glass.

\footnotetext{
* corresponding author: nejib.ihzaz@issatgb.rnu.tn
} 
Orthorhombic $R \mathrm{MnO}_{3}\left(\mathrm{o}-R \mathrm{MnO}_{3}, R=\mathrm{La}-\mathrm{Lu}, \mathrm{Y}\right)$ which exhibit a perovskite-like structure, have been potential research subjects due to their remarkable states resulting from the fine relationship between charge, spin, orbital, and lattice parameters [1-8]. Moreover, these materials can be epitaxially grown as thin films on different substrates. They exhibit compressive or tensile strains that are attributable to the lattice mismatch among the substrate and the corresponding layer. Hence, the strain produced by epitaxial growth is an efficient manner to obtain films with different transition temperatures, which may replay to different requirements for various scientific applications $[9,10]$. The bond length mismatch is adjusted in the orthorhombic $\mathrm{o}-\mathrm{RMnO} 3$ structure by rotation of the $\mathrm{MnO}_{6}$ octahedra resulting in bending of the equatorial and azimuthal $\mathrm{Mn}-\mathrm{O}-\mathrm{Mn}$ bond angles $\theta$ away from $180^{\circ}$ within all three crystallographic axes. These characteristics lead to the A-type antiferromagnetism (AFM) observed below the Neel temperature $\mathrm{T}_{\mathrm{N}}$ with ferromagnetic (FM) superexchange interactions in the planar section and AFM interactions within the c-axis perpendicular to a planar section for large ions $R=\mathrm{La}, \mathrm{Pr}, \mathrm{Nd}$, and $\mathrm{Sm}$ [11]. By decreasing the size of the $R$ ion $(\mathrm{R}=\mathrm{Eu}, \mathrm{Gd}, \mathrm{Tb}, \mathrm{Dy}$, and $\mathrm{Ho})$; i.e., with increased bending of the $\mathrm{Mn}-\mathrm{O}-\mathrm{Mn}$ bonds, many complex AFM structures have been surveyed. The complex structures with mixed FM/AFM superexchange interactions in the ab-plane have been accounted for as the next-nearestneighbor anisotropic relations. These relations occur from the closer distance and the less screened exchanges between the $\mathrm{Mn}$ ions [12]. The rare-earth manganite $\mathrm{NdMnO}_{3}$ belongs to the group of orthorhombically-distorted perovskites $R \mathrm{MnO}_{3}$ with the $\mathrm{GdFeO}_{3}$ symmetry. Among some perovskite-like oxides, the Nd-based samples exhibit a larger lattice distortion due to the little size of $\mathrm{Nd}$ atoms [13]. In thin-film form, the in-depth structural characterization and study of the magnetic behavior of Lacunar neodymium manganite were not addressed previously. Structural and magnetic results were only briefly published in the EPJ Web of Conferences [14] as a support of the interpretation of our structural and magnetic measurements. This article aims to provide complete information regarding this specimen.

\section{Experimental procedure}

NMO films were synthesized by injection MOCVD "band flash". An in-depth report of this experimental procedure has been detailed in Ref. [15]. The resulting study of the $\mathrm{Nd} / \mathrm{Mn}$ relative amount in the initial solution on the film composition was released by grown of lacunar perovskite-type manganese oxides $\mathrm{Nd}_{1-\mathrm{x}} \mathrm{MnO}_{3}$ films of about $200 \mathrm{~nm}$ thickness on 
STO with a different $\mathrm{Nd} / \mathrm{Mn}$ composition. The substrate temperature was at $680{ }^{\circ} \mathrm{C}$. The injections number is estimated at 1000 with a frequency of $1 / \mathrm{s}$, which is equivalent to a growth rate of $0.72 \mu \mathrm{m} / \mathrm{h}$. The as-deposited samples were then heat-treated, in an in situ manner, at $680{ }^{\circ} \mathrm{C}$ for 30 min under an $\mathrm{O}_{2}$ flow. The $\mathrm{Nd} / \mathrm{Mn}$ composition of the $\mathrm{Nd}_{1-x} \mathrm{MnO}_{3}$ films, measured by Wavelength Dispersive Spectroscopy (WDS) technique, versus the $\mathrm{Nd} / \mathrm{Mn}$ composition in the starting solution was performed. However, the oxygen elements in the layer/substrate system make quantitative resolve by WDS of the chemical composition very difficult. A linear relationship among the $\mathrm{Nd} / \mathrm{Mn}$ composition of precursor blend and that determined on the film has occurred. This investigation indicates the possibility to adjust the film composition. A scheme of the MOCVD reactor utilized in this project is detailed in figure 1(a). Sequence controlling of microliters droplets injected in the reactor (in a cold zone) on a moving tape is giving in figure 1(b). The composition of the solution required to attain a lacunar perovskite-type manganese oxides $\mathrm{Nd}_{0.88} \mathrm{MnO}_{3}$ (NMO) is 0.78 which means that the deposition proportion of the $\mathrm{Nd}(\text { thd })_{3}$ precursor is in small quantities compared to $\mathrm{Mn}$ (thd) $)_{3}$ within the deposition states used. The film has a thickness of about $200 \mathrm{~nm}$. X-ray diffraction (XRD) measurements were achieved with a BRUKER D8 advance research diffractometer $\theta / 2 \theta$ with a $\mathrm{Cu}$ anode $(\lambda=1.5406 \mathrm{~nm})$. Magnetization measurements were achieved using squid magnetometers (Quantum Design) in magnetic fields applied along or normal to the film surface. For every M-T curves, the film was cooled down to $10 \mathrm{~K}$ in zero magnetic fields, the magnetization measurements were achieved by reheating the film in an applied field of $0.05 \mathrm{~T}$ (zero-field cooling magnetization process noted $\mathrm{M}_{\mathrm{ZFC}}$ ). Afterward, the magnetization was obtained even as the sample was being cooled in the same magnetic field (field cooled magnetization process noted $\mathrm{M}_{\mathrm{FC}}$ ). Isothermal magnetizations $\mathrm{M}$ versus $\mathrm{H}$ curves were measured at $4 \mathrm{~K}$. Crystallographic images have been made with the program CrystalMaker [16].

\section{Results and discussion}

XRD pattern of the as-grown NMO film is shown in figure 2. It reveals that the film, in its first stage, is completely amorphous without any signal from microcrystals since apart from the STO (100) substrate reflections are apparent. Besides, just a large band in the spectrum is established. No significant turn in the observed XRD data occurred after the first stage of deposition at $680^{\circ} \mathrm{C}$. This examination indicates that the NMO layer has remained in an amorphous state after processing. After $24 \mathrm{~h}$ anneal at $900^{\circ} \mathrm{C}$, a new diffraction peak 
appeared. It is clear that the NMO film grows on the STO surface and exhibits a good crystalline state. Highly (100) textured and excellent OOP orientation of the NMO films is obtained. The stabilized phase results in the presence of minor secondary phases identified as $\mathrm{Mn}_{3} \mathrm{O}_{4}$ according to JCPDS cards 086-2337. To better understand and analyze this structural behavior, we return to an overview of both STO substrate and NMO bulk. STO crystallizes at room temperature in a cubic $\operatorname{Pm} \overline{3} \mathrm{~m}$ structure with $\mathrm{a}_{\text {STO }}=3.905 \AA$. Bulk ceramic sample NMO forms a distorted orthorhombic symmetry (Pnma space group) at room temperature; the orthorhombic lattice parameters are $\mathrm{a}=5.614 \AA, \mathrm{b}=7.607 \AA$, and $\mathrm{c}=5.407 \AA$ [17]. This preference of space group fits well with a convention for perovskite thin films. In these systems, the extended axis is marked the b-axis and is frequently chosen to be along to the substrate normal. Here we modify the basis of Ref. [18] so that the b-axis of Pnma maps to the c-axis (rather than b) of the cubic prototype specimenPm $\overline{3} \mathrm{~m}$. To compare with the pseudocubic perovskite structure of substrates, we introduce the small orthorhombic lattice defined as $a_{p}=a / \sqrt{2}, b_{p}=c / \sqrt{2}, c_{p}=b / 2$ [19]. We also define a pseudo-cubic symmetry with a mean lattice parameter $\mathrm{a}_{\mathrm{pc}}=\sqrt[3]{\mathrm{a}_{\mathrm{p}} \times \mathrm{b}_{\mathrm{p}} \times \mathrm{c}_{\mathrm{p}}}=3.864 \AA$ which is an almost ideal cubic perovskite. In general, an orthorhombic structure may be viewed conveniently as a cubic one:

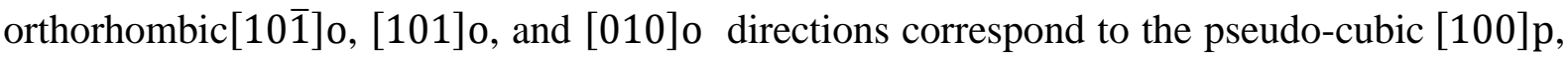
[010]p and [001]p directions, respectively. With these defined parameters, the NMO has to face the misfit strain defined as $\varepsilon_{\mathrm{m}}=\frac{\mathrm{a}_{\text {substrate }}-\mathrm{a}_{\mathrm{bulk}}}{\mathrm{a}_{\text {bulk }}}=1.10 \%$, where $\mathrm{a}_{\text {substrate }}\left(\mathrm{a}_{\mathrm{STO}}\right)$ and $\mathrm{a}_{\text {bulk }}\left(\mathrm{a}_{\mathrm{pc}}\right.$ in the pseudo-cubic notation) are respectively the strain-free lattice parameters of the substrate and the film. A diffraction process is observed along with the same sort of the plan $(h 00)$ of the pseudocubic perovskite cell as the substrate. The phase, therefore, appears to be textured in the direction perpendicular to the substrate plane. Moreover, the NMO diffraction peaks roughness and their emplacement close to the (h00) Bragg peaks of STO, indicates a good crystallinity and an OOP [100] texture. To describe more precisely the growth stabilization and the NMO structure, we study the (200) diffraction line of NMO (in the pseudo-cubic notation) which is well dissociated from the corresponding one of the substrate; scans are shown on figure 3. We can see strong film peaks and two very strong substrate overlapping peaks at right and left $2 \theta$ angle regions respectively. The clear dissimilarity of the film peaks corresponds to $\mathrm{Cu} \mathrm{k}_{\alpha 1}$ and $\mathrm{k}_{\alpha 2}$ radiation. From the peak positions, it is easy to find that the NMO film is grown with the IP tensile strain which in turn must produce a contraction of the layer OOP lattice distances. The (200) peak of the NMO 
film OOP parameter is around $2 \theta=47.1^{\circ} \pm 0.1^{\circ}$ and corresponding to $\mathrm{a}_{\perp}=3.8600 \AA \pm$ $0.005 \AA$. This value is a little higher than that observed for polycrystalline bulk samples at room temperature $\mathrm{a}_{\mathrm{pc}}$, so denoting a small contraction of OOP lattice distances and tensile stress over the IP lattice distance of the NMO. The real corresponding lattice mismatch is $\varepsilon_{\mathrm{m}}=1.16 \%$. The crystal structure of both $\mathrm{NMO}$ and STO, as well as a schematic

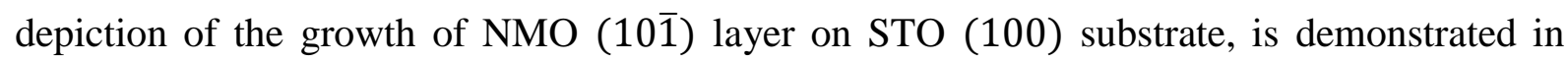

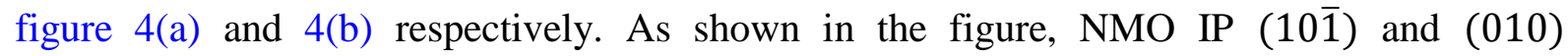
directions are aligned along with (100) and (001) directions of the cubic STO substrate. These structure models of NMO film and STO substrate are constructed by crystallographic simulation software CrystalMaker. Besides, the micro-structural distribution is equivalent to the results of the precedent TEM characterizations, occurred on the strain of other deposed films on STO systems [20-23]. Figure 5 shows the thermal evolution of field cooled $\mathrm{M}_{\mathrm{FC}}(\mathrm{T})$ and zero field cooled $\mathrm{M}_{\mathrm{ZFC}}(\mathrm{T})$ magnetization curves measured in $0.05 \mathrm{~T}$ of the OOP applied field. The exerted magnetic field $\mathrm{H}$ is expressed in SI units as $\mu_{0} \mathrm{H}$ all over this work. The data give evidence to ferromagnetic arrangement under the transition temperature $T_{C}=85 \mathrm{~K}$ defined as the inflection point of the derivative $\mathrm{dM}(\mathrm{T}) / \mathrm{dT}$ as shown in the inset (a) of figure 5, where the FC and ZFC measurements separate at $\mathrm{T}_{\text {irr }} \approx 48 \mathrm{~K}$ (inset (b) of figure 5). The $\left(\mathrm{T}_{\mathrm{C}}\right)$ magnetic transition temperature of the film is similar to the corresponding values of bulk compounds with little different nominal composition. In $\mathrm{NdMnO}_{3}$, magnetic ordering takes place at $\mathrm{T}=86 \mathrm{~K}$ [24] and $\mathrm{T}=88 \mathrm{~K}$ [25], whereas in $\mathrm{Nd}_{0.9} \mathrm{MnO}_{2.85}$ it occurs at $\mathrm{T}=85 \mathrm{~K}$ [26]. However, compared to bulk samples, the anisotropy of the magnetization in the thin film is less marked. Figure 6 shows the thermal evolution of field cooled $\mathrm{M}_{\mathrm{FC}}(\mathrm{T})$ curves measured in the OOP and IP directions in the $0.05 \mathrm{~T}$ applied field. Here, we remarked that magnetization $\mathrm{M}(\mathrm{T})$ in the OOP direction is twice as large as that in the IP direction. We think that the magnetic properties are related to both systems for controlling lattice mismatch and oxygen octahedral rotation mismatch. Several previous studies such as Monte-Carlo simulations and first-principles calculations founded on the double exchange form provided evidence that the oxygen octahedral rotation plays a more significant part than the superlattice dimension effect and might lead to canted magnetism [27,28]. Moreover, it is well known that the temperature transition $T_{C}$ depends on the electronic bandwidth $(W)[29,30]$ which is characterized by the overlap between the orbital $d$ of $\mathrm{Mn}$ and the orbital $p$ of $\mathrm{O}^{2-}$ and which can be empirically described by the equation [31]: 
Where $\theta_{M n-O-M n}$ and $d_{M n-O}$ are the $M n-O-M n$ bond angle and the $M n-O$ bond length respectively. The bond angle $\theta_{M n-O-M n}$ diminishes with the disappearance of the octahedral rotations contradicting the fact that ferromagnetism should be improved. A variation of the $\mathrm{e}_{\mathrm{g}}$ occupants owing to biaxial strain can reduce temperature transition $T_{C}$ depending on the distribution homogeneity of the given tetragonality near the interface between film and substrate. Because of the complexity of the factors involved in the evaluation of magnetic behavior such as crystal structure, stoichiometry, strain, temperature, growth conditions, etc, it is difficult to evaluate the precise origin of the magnetism behavior in our NMO/STO system. Figure 7 shows the magnetic hysteresis loop of the NMO thin film at $4 \mathrm{~K}$ in an IP and OOP field range 0 to $5 \mathrm{~T}$. by varying the applied magnetic field, a significant difference in the magnetization is observed. The OOP magnetization increased quickly than that measured in the IP direction. Moreover, it seems that the magnetization is not being saturated witch indicates the existence of the antiferromagnetic state. The remanent magnetizations $\left(M_{R}\right)$ and coercive fields $\left(\mathrm{H}_{\mathrm{C}}\right)$ were determined from the extended local $\mathrm{M}-\mathrm{H}$ hysteresis loops measured at $4 \mathrm{~K}$ (shown in inset Figure 7). These values are $\left(\mathrm{M}_{\mathrm{R}}=4.03 \times 10^{-4} \mathrm{emu}, \mathrm{H}_{\mathrm{C}}=0.031 \mathrm{~T}\right)$ and $\left(\mathrm{M}_{\mathrm{R}}=1.60 \times 10^{-4} \mathrm{emu}, \mathrm{H}_{\mathrm{C}}=0.025 \mathrm{~T}\right)$ for OOP and IP direction respectively. The curve M-H at T $=300 \mathrm{~K}$ shows similar paramagnetic behavior for both OOP and IP directions. These behaviors indicate the coexistence of an AFM and FM state in the film [32]. Taking into account all these observations, we can note that ZFC and FC magnetization curves of the NMO thin-film behaves differently. The ZFC magnetization increases progressively to a maximum value around a freezing temperature $T_{f}=53 \mathrm{~K}$ by increasing temperature $\left(\mathrm{T}_{\mathrm{f}}\right.$ is defined as $\left(\mathrm{M}_{\mathrm{FC}}-\mathrm{M}_{\mathrm{ZFC}}\right) / \mathrm{M}_{\mathrm{ZFC}}$, which becomes different from zero, indicating the onset of a freezing process) and then decreases monotonously. On the contrary, the FC magnetization decreases progressively with increasing temperature. A clear bifurcation of the FC and ZFC with a lambda shaped-like has been observed. These phenomena are collectively treated before as spin-glass system (SG) behaviors, where $T_{\text {irr }}$ is generally close to but slightly below $\mathrm{T}_{\mathrm{f}}$ [33-35]. Also, although in the cluster glass (CG) systems, $\mathrm{T}_{\text {irr }}$ is usually higher than $\mathrm{T}_{\mathrm{f}}$, [36,37]. Here, we can still exclude the superparamagnetic (SPM) system where the magnetization after FC increases rapidly and monotonously with the lowering of the temperature [38]. Creating deficits at the A-site sub-lattices induces a dilution of the magnetic exchange between $\mathrm{Mn}^{3+}$ and $\mathrm{Mn}^{4+}$ ions via oxygen, a disorder, and a change in the $\mathrm{Mn}^{3+}-\mathrm{O}^{2-}-$ 
$\mathrm{Mn}^{4+}$ bond angle in the system. The balance of competing for superexchange pathways between the transition metals matrix $\mathrm{Mn}^{3+} / \mathrm{Mn}^{4+}$ state is modified which supports the most likely scenario suggesting a spin-glass behavior with long-range ferromagnetic order $\left(\mathrm{Mn}^{3+}-\right.$ $\left.\mathrm{O}^{2-}-\mathrm{Mn}^{4+}\right)$ co-exists in NMO thin films $[39,40]$.

\section{Conclusion}

In summary, we grew high-quality growth NMO (101) thin film on STO (100) substrate using liquid-injection MOCVD. Structural analysis shows that the grown film was amorphous, but after annealing at $900^{\circ} \mathrm{C}$ in the air for $24 \mathrm{~h}$, it changed to the crystalline phase. The NMO thin film showed ferromagnetic ordering with a transition temperature $T_{C}=85 \mathrm{~K}$. ZFC and FC magnetization curves split with a sharp cusp in the ZFC curves at a low temperature around the freezing temperature $\mathrm{T}_{\mathrm{f}}=53 \mathrm{~K}$ and an irreversible temperature $\mathrm{T}_{\text {irr }}=$ $48 \mathrm{~K}$, indicating spin-glass behavior with long-range ferromagnetic order $\left(\mathrm{Mn}^{3+}-\mathrm{O}^{2-}-\mathrm{Mn}^{4+}\right)$ and an OOP magnetic easy axis.

\section{References}

[1] M. Tachibana, T. Shimoyama, H. Kawaji, T. Atake, and E. Takayama-Muromachi, Phys. Rev. B 75144425 (2007).

[2] T. Kimura, S. Ishihara, H. Shintani, T. Arima, K. T. Takahashi, K. Ishizaka, and Y. Tokura, Phys. Rev. B 68060403 (2003).

[3] T. Goto, T. Kimura, G. Lawes, A. P. Ramirez, and Y. Tokura, Phys. Rev. Lett. 92, 257201 (2004).

[4] T. Kimura, T. Goto, H. Shintani, K. Ishizaka, T. Arima, and Y. Tokura, Nature 426 (2003).

[5] T. Kimura, G. Lawes, T. Goto, Y. Tokura, and A. P. Ramirez, Phys. Rev. B 71224425 (2005).

[6] V. Yu. Pomjakushin, M. Kenzelmann, A. Dönni, A. B. Harris, T. Nakajima, S.

Mitsuda, M. Tachibana, L. Keller, J. Mesot, H. Kitazawa, and E. TakayamaMuromachi, New J. Phys. 11, 043019 (2009).

[7] S. Ishiwata, Y. Kaneko, Y. Tokunaga, Y. Taguchi, T.-h. Arima, and Y. Tokura, Phys. Rev. B 81100411 (2010).

[8] D. Okuyama, S. Ishiwata,Y. Takahashi, K.Yamauchi, S. Picozzi, K. Sugimoto, H. Sakai, M. Takata, R. Shimano, Y. Taguchi, T. Arima, and Y. Tokura, Phys. Rev. B 84 
054440 (2011).

[9] D. G. Schlom, L.-Q. Chen, C. J. Fennie, V. Gopalan, D. A. Muller, X. Pan, R. Ramesh, and R. Uecker, MRS Bull. 39 118-130 (2014).

[10] B. Yildiz, MRS Bull. 39 147-156 (2014).

[11] J.B. Goodenough, Magnetism and the chemical bond, Wiley, New York,1963.

[12] J. A. Alonso, M. J. Martínez-Lope, M. T. Casais And M. T. Fernández-Díaz, Inorg. Chem. 39( 5) 917-923 (2000).

[13] A. Nandy, A. Roychowdhury, D. Das, and S. K. Pradhan, Powder Technol., 254538 547 (2014).

[14] N. Ihzaz, M. Boudard, L. Rapenne, H. Roussel, S. Pignard and M. Oumezzine, EPJ Web of Conferences 2900024 (2012).

[15] Nejib Ihzaz, Mohamed Oumezzine, Jens Kreisel, Henri Vincent, and Stephane Pignard, Chem. Vap. Deposition 14 111-114 (2008).

[16] D. Palmer, CrystalMaker Software Version 2.7.7, CrystalMaker Software Ltd., 2013.

[17] Elaine T. Maguire, Alison M. Coats, Janet M. S. Skakle and Anthony R.West, J. Mater. Chem., 9 1337-1346 (1999).

[18] Christopher J. Howard and Harold T. Stokes, Acta Cryst. B54 $782-789$ (1998).

[19] N. W. Thomas, Acta Crystallogr. B 52 (1996) 16-31.

[20] Zhipeng Li, Dongsheng Song, Rong Yu, Binghui Ge, Zhenyu Liao, Yueliang Li, Shuai Dong, and Jing Zhu, ACS Appl. Mater. Interfaces 824192-24197 (2016).

[21] Weizong Xu, Preston C. Bowes, Everett D. Grimley, Douglas L. Irving, and James M. LeBeau, Appl. Phys. Lett. 109 201601(2016).

[22] Shaobo Cheng, Changsong Xu, Shiqing Deng, Myung-Geun Han, Shanyong Bao, Jing Ma, Cewen Nan, Wenhui Duan, Laurent Bellaiche, Yimei Zhu, and Jing Zhu, Sci. Adv. 4 eaar4298 (2018).

[23] Weiwei Li, Bonan Zhu, Qian He, Albina Y. Borisevich, Chao Yun, Rui Wu, Ping Lu, Zhimin Qi, Qiang Wang, Aiping Chen, Haiyan Wang, Stuart A. Cavill, Kelvin H. L. Zhang, and Judith L. MacManus-Driscoll, Adv. Sci. 71901606 (2020).

[24] K. Nakamura, J. Solid State Chem. 173, 299 (2003).

[25] J. Hemberger, M. Brando, R. Wehn, V. Yu. Ivanov, A. A. Mukhin, A. M. Balbashov, and A. Loidl, Phys. Rev. B: B 69064418 (2004).

[26] I.O. Troyanchuk, Physics of the Solid State, 48(5) 898-903 (2006). 
[27] Li Yin, Qian Zhang, Wenbo Mi, and Xiaocha Wang J. Appl. Phys. 120, 165303 (2016).

[28] Xiaofang Zhai, Long Cheng, Yang Liu, Christian M. Schlepütz, Shuai Dong, Hui Li, Xiaoqiang Zhang, Shengqi Chu, Lirong Zheng, Jing Zhang, Aidi Zhao, Hawoong Hong, Anand Bhattacharya, James N. Eckstein Changgan Zeng, Nat. Commun. 5, 4283 (2014).

[29] Coey, J. M. D., Viret, M., Molnar, S. v. Adv. Phys. 48, 167-293 (1999).

[30] Garcia-Munoz, J. L., Fontcuberta, J., Suaaidi, M. \& Obradors, X, J. Phys.: Condens. Matter 8, L787-L793 (1996).

[31] Cui, C. , Tyson, T. A., Appl. Phys. Lett. 84, 942 (2004).

[32] S Biswas, S Pal and E Bose, Indian Journal of Physics, 88(10) 1045-1049 (2014).

[33] J. Alonso, M. L. Fdez-Gubieda, J. M. Barandiar_an, A. Svalov, L. Fern_andez Barqu_1n, D. Alba Venero, and I. Orue, Phys. Rev. B 82, 054406 (2010).

[34] S. Karmakar, S. Taran, E. Bose, B. K. Chaudhuri, C. P. Sun, C. L. Huang, and H. D. Yang, Phys. Rev. B 77, 144409 (2008).

[35] T. D. Thanh, D. H. Manh, T. L. Phan, P. T. Phong, L. T. Hung, N. X. Phuc, and S. C. Yu, J. Appl. Phys. 115, 17B504 (2014).

[36] X. G. Li, X. J. Fan, G. Ji, W. B. Wu, K. H. Wong, C. L. Choy, and H. C. Ku, J. Appl. Phys. 85, 1663 (1999).

[37] X. H. Huang, J. F. Ding, Z. L. Jiang, Y. W. Yin, Q. X. Yu, and X. G. Li, J. Appl. Phys. 106, 083904 (2009)

[38] X. Battle and A. Labarta, J. Phys. D: Appl. Phys. 35 R15 (2002).

[39] J. R. L. De Almeida and D. J. Thouless, J. Phys. A 11, 983 (1978).

[40] M. Gabay and G. Toulouse, Phys. Rev. Lett. 47, 201 (1981). 
Figure 1. (a) Scheme of the liquid source injection MOCVD reactor used in this study, (b) injector and a Sequence controlling of microliters droplets injected in the reactor (in a cold zone) .

Figure 2. X-ray diffraction patterns of as-deposited NMO thin films for $20^{\circ}<2 \theta<80^{\circ}$.

Figure 3. X-ray diffraction patterns of as-deposited NMO thin films for $44^{\circ}<2 \theta<50^{\circ}$.

Figure 4. Schematic diagram showing: (a) Crystal structure of NMO, Crystal structure of STO and Schematic representation of the (101) epitaxial growth of orthorhombic LMO thin

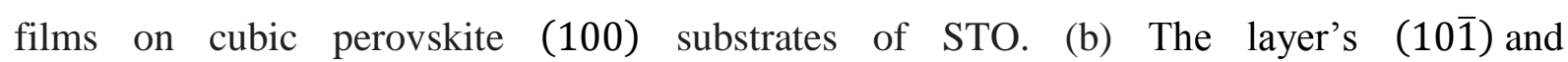
(010) directions are aligned along (100) and (001)directions of the substrate.

Figure 5. Temperature dependence of the field cooled $\mathrm{M}_{\mathrm{FC}}(\mathrm{T})$ and zero field cooled $\mathrm{M}_{\mathrm{ZFC}}(\mathrm{T})$ magnetization curves measured in $0.05 \mathrm{~T}$ of the out-of-plane applied field. Inset (a) shows the $\mathrm{dM} / \mathrm{dT}$ curve for determining $\mathrm{T}_{\mathrm{C}}$. Inset (b) shows the method described in the text to calculate the onset of weak and strong irreversibility temperatures from the irreversible magnetization (i.e., $\left.\left(\mathrm{M}_{\mathrm{FC}}-\mathrm{M}_{\mathrm{ZFC}}\right) / \mathrm{M}_{\mathrm{ZFC}}\right)$.

Figure 6. Thermal evolution of field cooled $\mathrm{M}_{\mathrm{FC}}(\mathrm{T})$ curves measured in the out-of-plane (OOP) and in-plane (IP) $0.05 \mathrm{~T}$ applied field.

Figure 7. $\mathrm{M}(\mathrm{H})$ hysteresis loops of NMO film at $4 \mathrm{~K}$ and $300 \mathrm{~K}$ along OOP and IP directions. The inset shows in detail the low field region. 


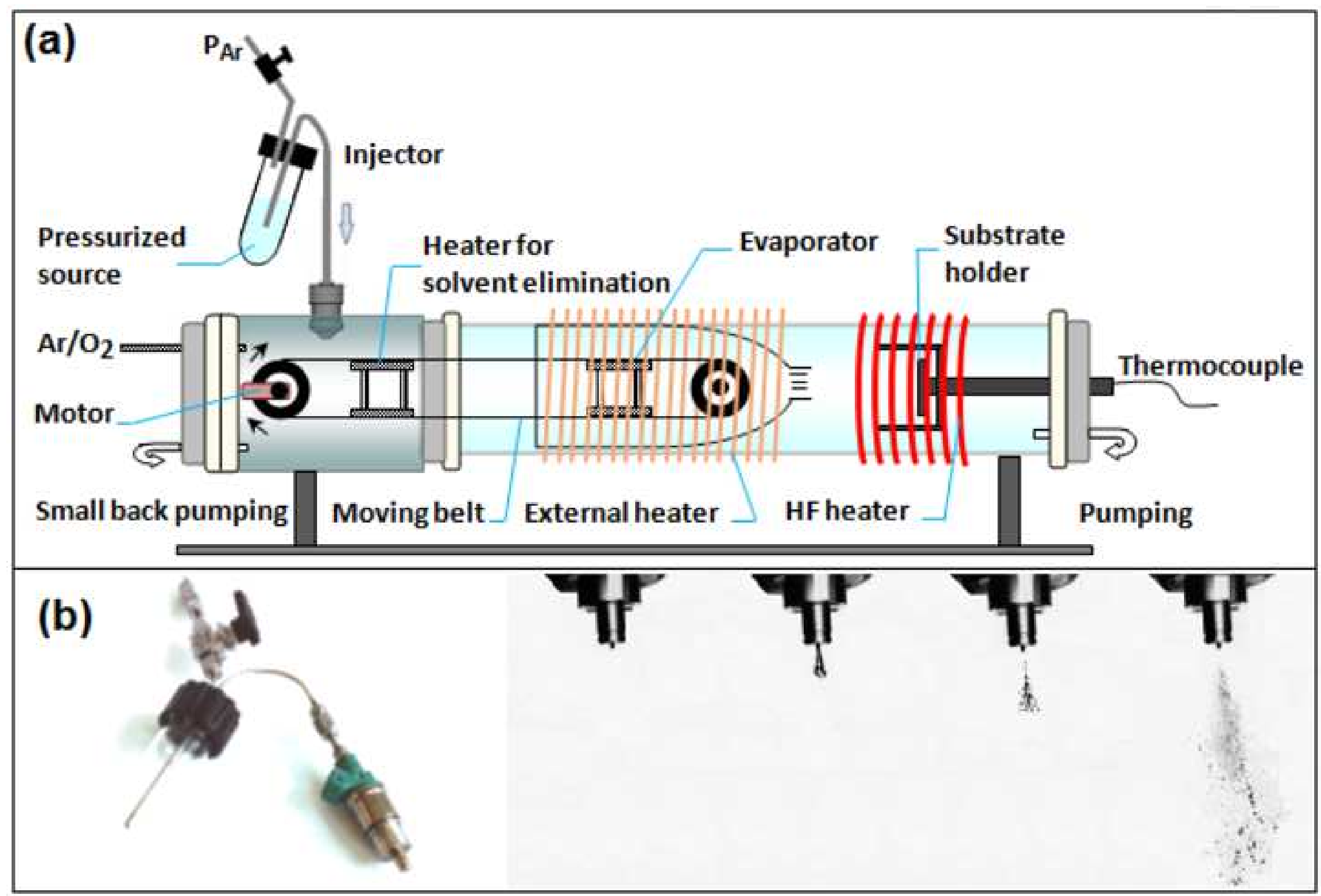




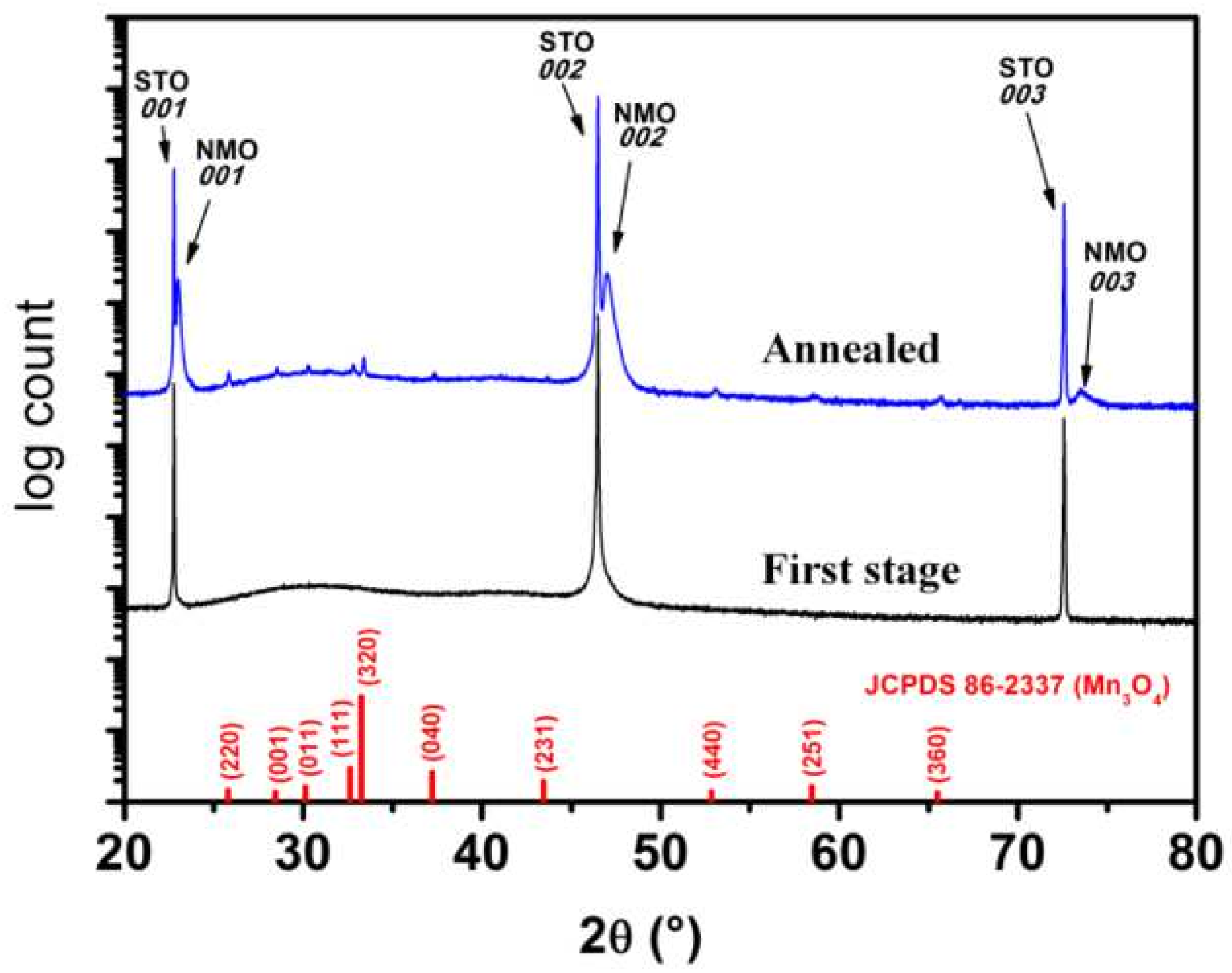




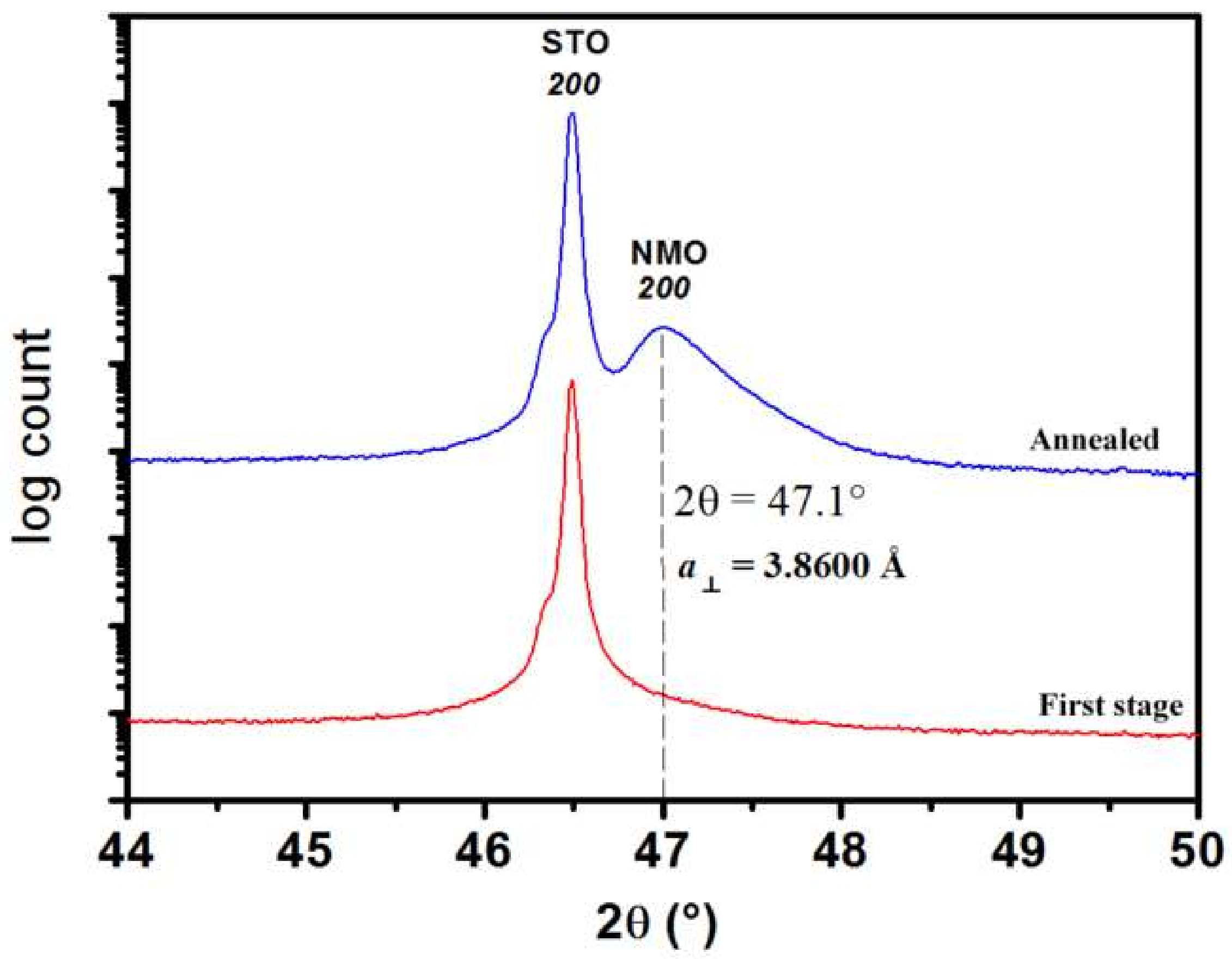



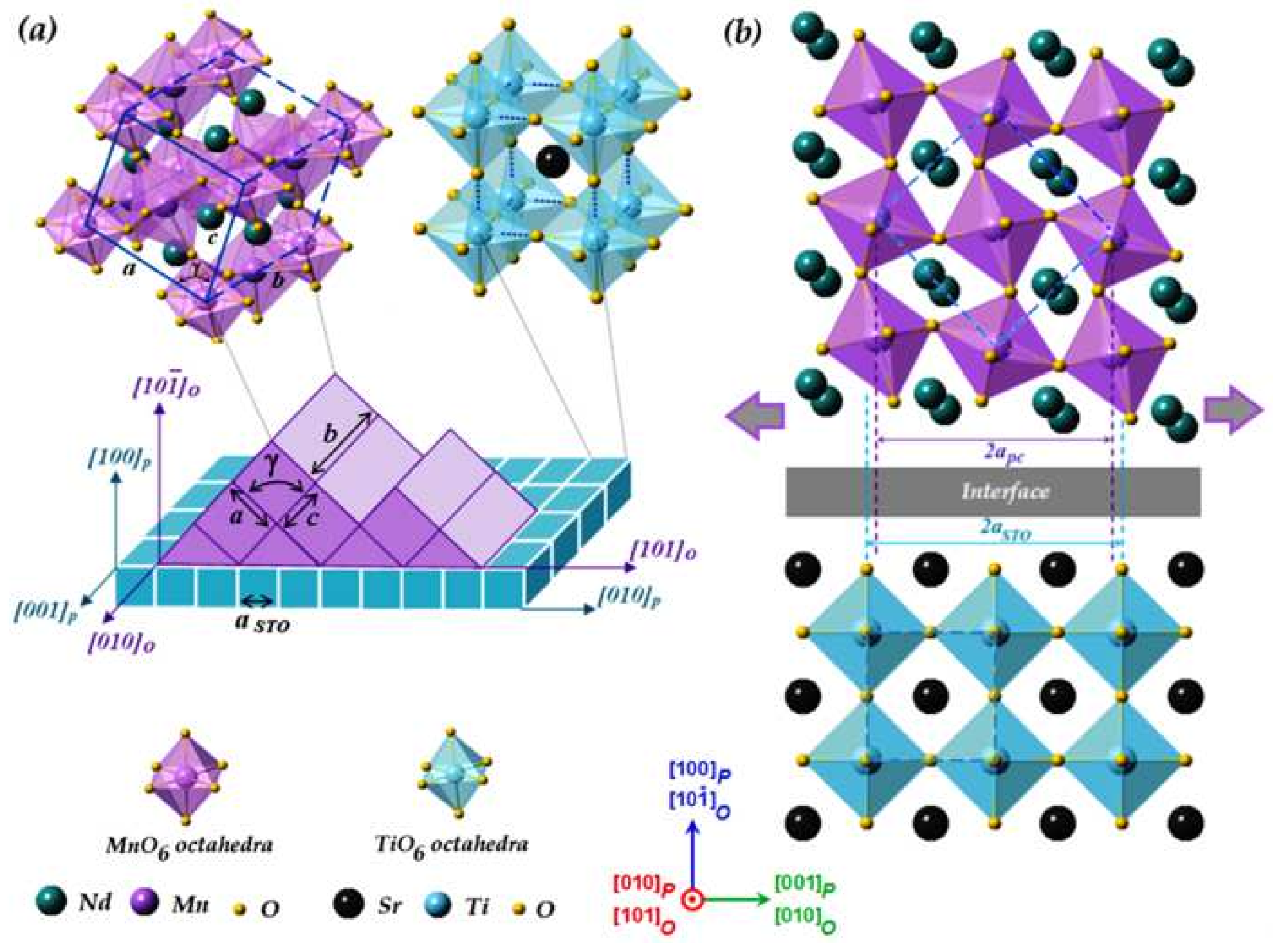

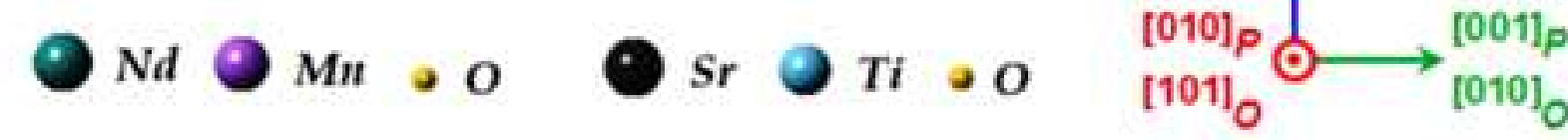




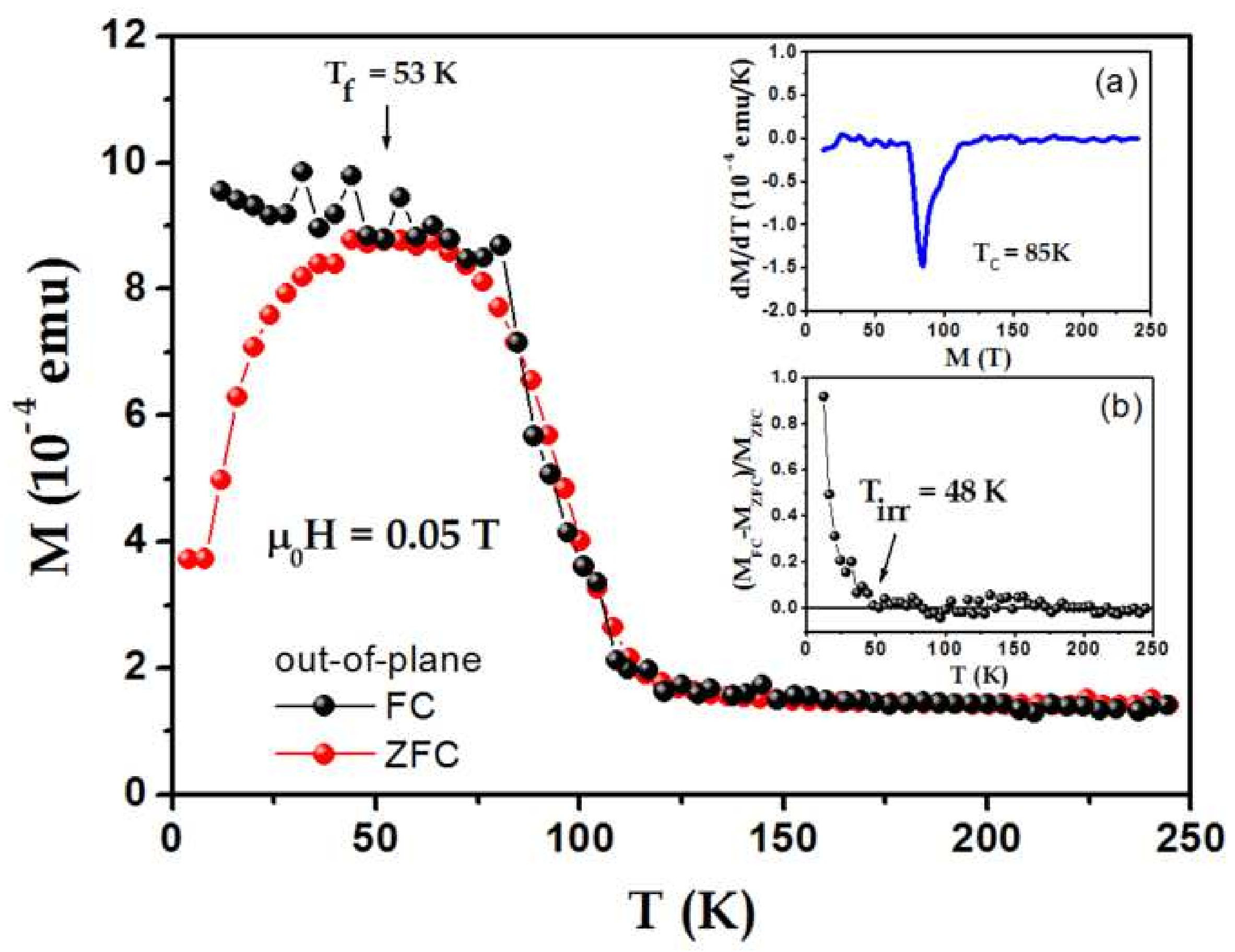




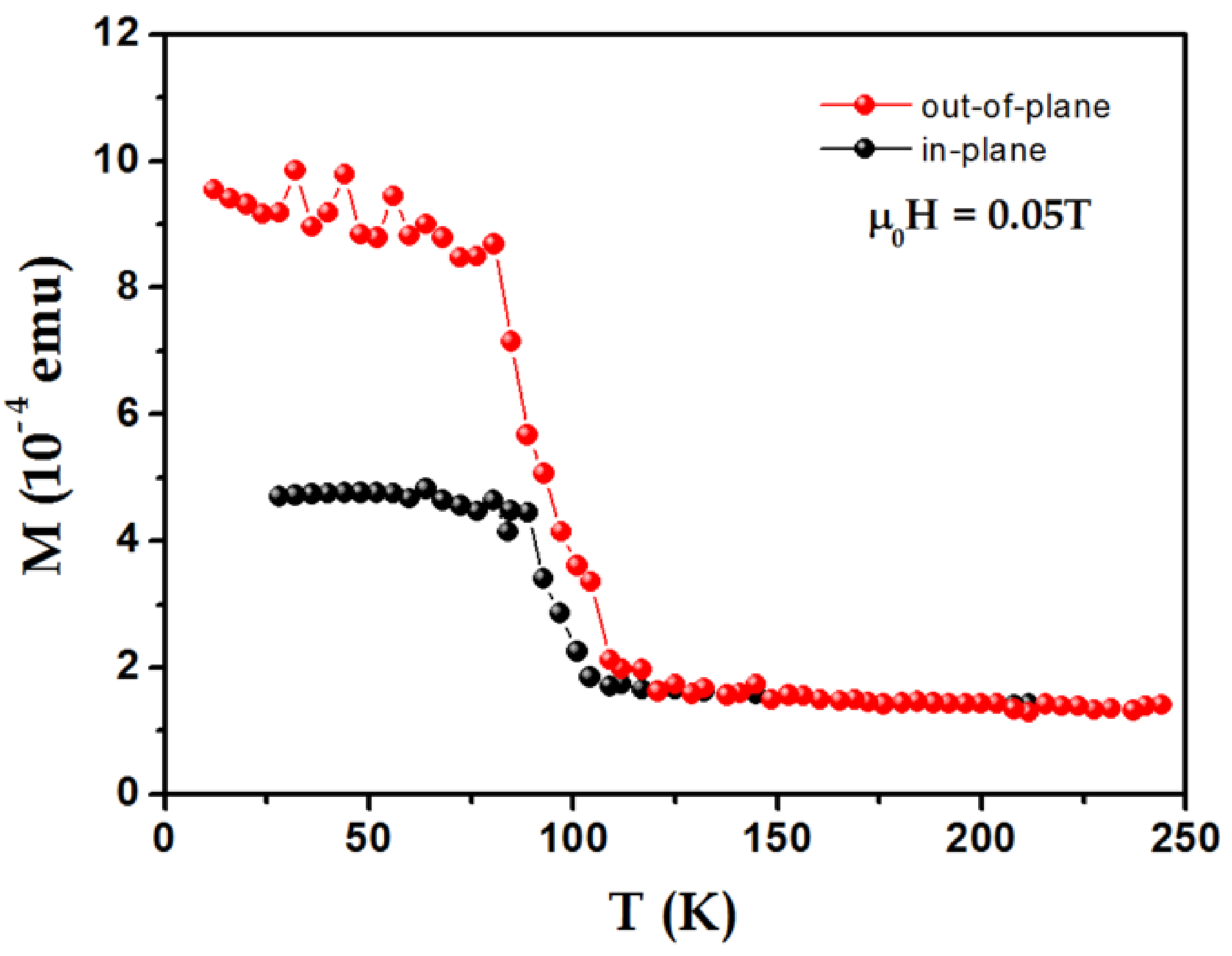




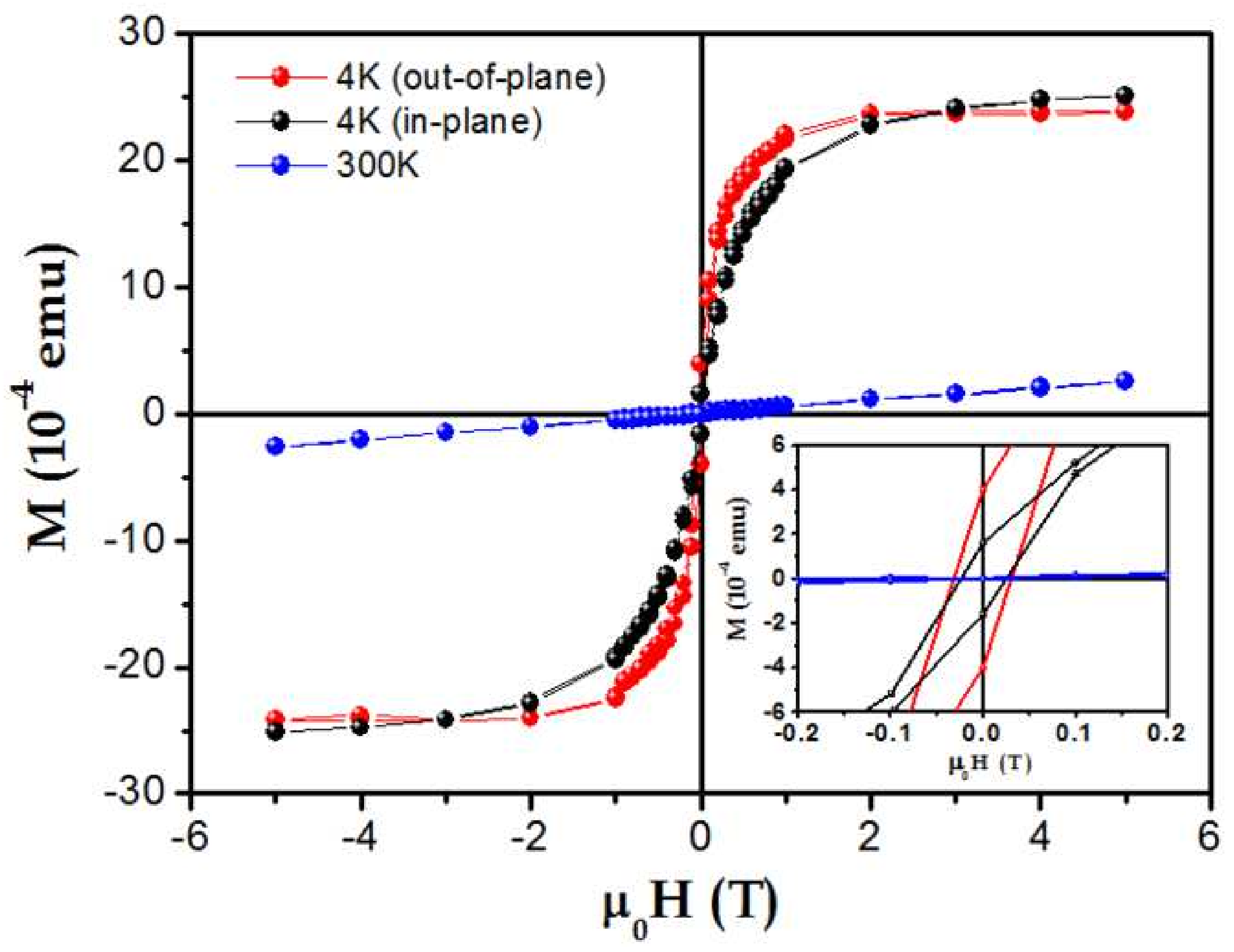

